\title{
CX3CL1/fractalkine enhances prostate cancer spinal metastasis by activating the Src/FAK pathway
}

\author{
PENG LIU, YUN LIANG, LIBO JIANG, HOULEI WANG, SHENGXING WANG and JIAN DONG \\ Department of Orthopedic Surgery, Zhongshan Hospital, Fudan University, Shanghai 200032, P.R. China
}

Received May 2, 2018; Accepted June 29, 2018

DOI: 10.3892/ijo.2018.4487

\begin{abstract}
Chemokines serve important roles in the development of cancer. C-X3-C motif chemokine ligand 1 (CX3CL1) has been demonstrated to promote metastases in different types of tumors. The authors' previous studies demonstrated that the CX3CL1 (also termed fractalkine)/steroid receptor coactivator (Src)/focal adhesion kinase (FAK) signaling pathway is associated with spinal metastasis. In the present study, it was observed that CX3CL1/C-X3-C motif chemokine receptor 1 (CX3CR1) was overexpressed in prostate cancer tissues with spinal metastasis compared with primary tumors. Overexpression of CX3CR1 induced cell proliferation, migration and invasion, and inhibited cellular apoptosis. However, repression of CX3CR1 reduced cell proliferation, migration and invasion, and increased cellular apoptosis. In addition, the Src/FAK pathway was activated by CX3CL1, which depends on the Tyr992 residue of epidermal growth factor receptor (EGFR) for phosphorylation. The inhibitors of these kinases repressed the cell migration induced by CX3CL1 or CX3CR1 overexpression. Furthermore, overexpression of CX3CR1 induced the spinal metastasis of prostate cancer in an in vivo mouse model. Therefore, CX3CL1 and its regulation of the EGFR, Src and FAK pathways may be potential targets for the early prevention of spinal metastasis in prostate cancer.
\end{abstract}

\section{Introduction}

Bone is the most common site of metastasis in prostate cancer (1). Approximately two-thirds of all bone metastases are located in the spine (2). A reported one-third of prostate metastases to the spine become symptomatic as spinal cord compression or mechanical instability (3-5). Undoubtedly,

Correspondence to: Dr Jian Dong, Department of Orthopedic Surgery, Zhongshan Hospital, Fudan University, 180 Fenglin Road, Shanghai 200032, P.R. China

E-mail: dong.jian@zs-hospital.sh.cn

Key words: spinal metastasis, prostate cancer, C-X3-C motif chemokine ligand 1/C-X3-C motif chemokine receptor 1, epidermal growth factor receptor/steroid receptor coactivator/focal adhesion kinase pathway spinal metastasis increases the risk of pathological fracture, spinal cord compression and intractable cancer-induced bone pain, and decreases survival (6). A previous study reported that the 5-year survival rate approaches $100 \%$ in patients with localized prostate cancer; however, this rate drops to $33 \%$ in patients with distant metastases (7). Therefore, gaining a better understanding of the mechanism by which prostate cancer metastasizes to distant organs, including the spine, is essential to the development of targeted drugs and the improvement of patient survival.

Chemokines belong to the cytokine family, and are small secreted proteins composed of 70-100 amino acids (8). Recent studies have demonstrated that chemokines and their receptors are involved in a variety of physiological and pathological processes, including cell growth, differentiation, apoptosis, tumor growth and metastasis $(9,10)$. C-X3-C motif chemokine ligand 1 (CX3CL1) has been demonstrated to promote metastases in different types of tumors (11-13). Unlike other chemokines, CX3CL1 (also termed fractalkine) has a unique receptor, $\mathrm{C}-\mathrm{X} 3-\mathrm{C}$ motif chemokine receptor 1 (CX3CR1). CX3CL1 exists in a membrane-bound and a soluble form. The membrane-bound form promotes adhesion between tumor cells and endothelial cells, while the soluble form recruits cells that express CX3CR1 (14). Human prostate cancer cells express CX3CR1, and the association between CX3CL1 and CX3CR1 regulates cell adhesion, migration and survival (15). Furthermore, the interaction between CX3CL1 and its receptor $\mathrm{CX} 3 \mathrm{CR} 1$ in the bone marrow serves a crucial role in directing circulating prostate cancer cells to the bone via androgen receptors (16). Recent work using high-throughput microarrays demonstrated that CX3CL1 is associated with spinal metastasis in different cancer types (17). However, the underlying mechanism of CX3CL1 in spinal metastasis remains unclear.

The results of the present study demonstrated that CX3CL1/CX3CR1 was overexpressed in prostate cancer tissues with spinal metastasis compared with primary tumors. Overexpression of CX3CR1 increased cell proliferation, migration and invasion. It was further observed that the epidermal growth factor receptor (EGFR)/Src/FAK pathway was activated by $\mathrm{CX} 3 \mathrm{CL} 1 / \mathrm{CX} 3 \mathrm{CR} 1$. The inhibitors of these kinases reversed the cell migration promoted by CX3CL1/CX3CR1. Notably, overexpression of CX3CR1 induced the spinal metastasis of prostate cancer in vivo. Thus, the present data provide a novel signaling pathway that merits further study to 
examine the mechanism of CX3CL1/CX3CR1-induced spinal metastasis in prostate cancer.

\section{Materials and methods}

Clinical specimens. A total of 48 clinical specimens were obtained from the Department of Orthopedic Surgery, Zhongshan Hospital, Fudan University (Shanghai, China) between December 2014 and December 2017. These included 12 cases of prostate carcinoma with spinal metastasis (male, 12; age, $67.00 \pm 10.43$ years), 12 cases of primary spinal tumor (male/female, 8/4; age, $52.75 \pm 16.54$ years), 12 samples of healthy vertebrae (male/female, $7 / 5$; age, $51.92 \pm 12.80$ years) and 12 samples of healthy limb bone tissue (fractures; male/female, 8/4; age, 53.08 \pm 15.81 years). Patients who had received treatment prior to surgery or for whom the pathological diagnosis was unclear were excluded. All the clinical samples were approved by the Ethics Committee of Zhongshan Hospital, Fudan University (no. Y2014-185). These included 12 cases of prostate carcinoma with spinal metastasis, 12 cases of primary spinal tumor, 12 samples of healthy vertebrae and 12 samples of healthy limb bone tissue (fractures). The diagnoses of the tumors were verified based on postoperative pathological reports. All the samples that included primary prostate tumor tissues, prostate tumor tissues with spinal metastasis and paracancerous tissues were collected during surgery and placed in a liquid nitrogen tank. The samples were stored at $-80^{\circ} \mathrm{C}$ until further analysis. Blood samples were collected and centrifuged for $10 \mathrm{~min}(1,000 \mathrm{x} \mathrm{g}$ at room temperature), and the supernatant was used for ELISA analysis. Informed consent was provided by the patients prior to surgery.

Cell lines, lentiviruses and small interfering (si)RNA. A total of four prostate cancer cell lines (PC-3, VCaP, LNCaP and 22RV1) and one healthy prostate epithelial cell line (RWPE-1) were obtained from the Chinese Academy of Sciences cell bank (Shanghai, China). PC-3 cells were cultured in F-12 (cat. no. 21127022; Gibco; Thermo Fisher Scientific, Inc., Waltham, MA, USA); VCaP cells were cultured Dulbecco's modified Eagle's medium (DMEM; cat. no. 10569044; Gibco; Thermo Fisher Scientific, Inc.); LNCaP and 22RV1 cells were cultured in RPMI-1640 media (cat. no. 61870044; Gibco; Thermo Fisher Scientific, Inc.); and RWPE-1 cells were cultured in Keratinocyte-SFM medium (cat. no. 10725018; Invitrogen; Thermo Fisher Scientific, Inc.). These four complete media all contained $10 \%$ fetal bovine serum (FBS; cat. no. 10099-141; Gibco; Thermo Fisher Scientific, Inc.) and $1 \%$ penicillin and streptomycin $(100 \mathrm{U} / \mathrm{ml}$ penicillin and $100 \mathrm{U} / \mathrm{ml}$ streptomycin; cat. no. B557; Invitrogen; Thermo Fisher Scientific, Inc.). Cells were incubated in a humidified atmosphere containing $5 \% \mathrm{CO}_{2}$ at $37^{\circ} \mathrm{C}$. The $\mathrm{CX} 3 \mathrm{CR} 1$-overexpressing lentiviruses were purchased from Shanghai GeneChem Co., Ltd. (Shanghai, China), and the CX3CR1 and control siRNAs were purchased from Shanghai GenePharma Co., Ltd. (Shanghai, China). The siRNA sequence targeting CX3CR1 was: 5'-AAAAATCAA CGTGGACTGAGC-3'. The negative control sequence was: 5'-UUCUCCGAACGUGUCACGUTT-3'.

Treatment with CX3CL1 and transfection. PC-3 and VCaP cells were treated with CX3CL1 (100 nM; R\&D Systems, Inc.,
Minneapolis, MN, USA) for $30 \mathrm{~min}$ at $37^{\circ} \mathrm{C}$. For the lentiviral infections, PC-3 and VCaP cells $\left(2 \times 10^{5}\right)$ were seeded onto 6 -well plates. This was followed the next day by infection with either control or CX3CR1-overexpressing lentiviruses in the presence of polybrene (final concentration, $6 \mu \mathrm{g} / \mu \mathrm{l}$ ). A total of 2-3 days following infection, cells were subcultured and selected with $5 \mu \mathrm{g} / \mathrm{ml}$ puromycin. For the siRNA transfections, PC-3 and VCaP cells $\left(2 \times 10^{5}\right)$ were seeded onto 6-well plates, and $100 \mathrm{nM}$ of either control siRNA or CX3CR1 siRNA was transfected using Lipofectamine ${ }^{\circledR} 2000$ reagent (Invitrogen; Thermo Fisher Scientific, Inc.). At 48 h post-transfection, the cells were recultured for 0-5 days for the different detection procedures. All the cells were divided into six groups, namely the blank control (CON), CX3CL1 group, overexpression negative control [NC (OE)], overexpression (OE), knockdown $\mathrm{NC}$ [NC (KD)] and knockdown (KD) groups, for cell functional analysis.

Cell counting kit-8 (CCK-8) assays. VCaP and PC-3 cell suspensions were added to a 96 -well plate at a density of $1 \times 10^{4}$ cells $/ \mathrm{ml}$. A total of $10 \mu \mathrm{l}$ CCK- 8 solution (Dojindo Molecular Technologies, Inc., Kumamoto, Japan) was added to each well at the same time every day for 5 days. Finally, the absorbance at $450 \mathrm{~nm}$ was measured using a microplate reader (Thermo Fisher Scientific, Inc.) following a $2 \mathrm{~h}$ incubation period prior to detection.

Cellular apoptosis assay. A total of $5 \times 10^{5} \mathrm{VCaP}$ and $\mathrm{PC}-3$ cells were harvested and the cells were suspended in $500 \mu \mathrm{l}$ binding buffer [cat. no. 70-AP101-100-BB; Hangzhou Multi Sciences (Lianke) Biotech Co., Ltd., Hangzhou, China]. Subsequently, $5 \mu$ l Annexin V-fluorescein isothiocyanate and $10 \mu \mathrm{l}$ propidium iodide (Invitrogen; Thermo Fisher Scientific, Inc.) was added to cells and the cells were incubated at $37^{\circ} \mathrm{C}$ for $15 \mathrm{~min}$ in the dark. Flow cytometry analysis was performed (BD FACSCalibur; BD Biosciences, Franklin Lakes, NJ, USA) to detect cellular apoptosis. The results were analyzed using FlowJo software (version 10.0; FlowJo, LLC, Ashland, OR, USA).

Scratch wound assay. VCaP cells were seeded onto 6-well plates. When cells had grown over the entire bottom of the well in a monolayer, a $100 \mu \mathrm{l}$ pipette tip was used to produce a scratch in the wells. A total of $2 \mathrm{ml}$ DMEM without FBS was added to maintain the culture. CX3CL1 (100 nM) was used in all groups except the CON group. The alteration in distance between the two edges of the wound was observed through optical microscopy (Olympus-IX51; Olympus Corporation, Tokyo, Japan) at time points of 0 and $96 \mathrm{~h}$ and x100 magnification.

Transwell assays. A $24-w e l l$ Transwell plate $(8-\mu \mathrm{m}$ pore size; Corning Incorporated, Corning, NY, USA) was selected for the present study. The upper compartment of the polycarbonate filter was coated with Matrigel (Sigma-Aldrich; Merck KGaA, Darmstadt, Germany). The Matrigel matrix (5 mg/ml; $100 \mu \mathrm{l}$ ) formed a continuous thin layer following drying for $1 \mathrm{~h}$ at $37^{\circ} \mathrm{C}$. The PC-3 cells $\left(1 \times 10^{5}\right)$ were seeded onto the upper chamber containing $100 \mu \mathrm{l} \mathrm{FBS-free} \mathrm{F12} \mathrm{culture} \mathrm{medium,} \mathrm{and} 600 \mu \mathrm{l}$ F12 culture medium containing 20\% FBS was added to the 
lower chamber. CX3CL1 (100 nM) was used in all groups, except the CON group. The whole culture plate was incubated for $24 \mathrm{~h}$. Finally, the lower surface of the upper chamber was observed following $4 \%$ paraformaldehyde fixation for $20 \mathrm{~min}$ and $0.1 \%$ crystal violet staining for $15 \mathrm{~min}$ at room temperature.

Signaling pathway analysis. The Ingenuity Pathway Analysis (IPA) database (https://www.qiagenbioinformatics.com/products/ingenuity-pathway-analysis) was used to further predict the potential factors involved in the CX3CL1-associated Src/FAK signaling pathway in prostate cancer.

Western blot analysis. The whole cell lysate of tissues and cells was harvested in lysis buffer (cat. no. P0013; Beyotime Institute of Biotechnology, Haimen, China) containing a phosphorylase inhibitor cocktail (Abcam, Cambridge, MA, USA) and phenylmethanesulfonyl fluoride (Beyotime Institute of Biotechnology). Additionally, when EGFR inhibitor afatinib, Src inhibitor bosutinib, and FAK inhibitor PF-562271 (Selleck Chemicals, Houston, TX, USA) were used, the cells were pretreated in accordance with previous studies (18-21). Bromophenol blue 2X (Amresco, LLC, Solon, OH, USA) was added as a loading buffer. An equal amount $(20 \mu \mathrm{g}$; bicinchoninic acid assay) of each sample was electrophoresed on an 8-12\% SDS-PAGE gel and transferred onto polyvinylidene fluoride membranes using an electrotransfer system (Bio-Rad Laboratories, Inc., Hercules, CA, USA). Subsequently, the membranes were incubated with specific antibodies, following blocking with $5 \%$ skimmed milk powder for $2 \mathrm{~h}$ at room temperature. The membranes were incubated with specific antibodies, including CX3CL1 (1:1,000; cat. no. ab25088), CX3CR1 (1:1,000; cat. no. ab8021; Abcam), Rho-associated protein kinase 1 (ROCK1; 1:2,000; cat. no. ab45171), matrix metalloproteinase-9 (MMP-9; 1:1,000; cat. no. ab73734) (all from Abcam), FAK (1:1,000; cat. no. 3285), phosphorylated (p)-FAK (1:1,000; cat. no. 3281), Src (1:1,000; cat. no. 2108), p-Src (1:1,000; cat. no. 6943), EGFR (1:1,000; cat. no. 5616), p-EGFR (1:1,000; cat. no. 2235) [Cell Signaling Technology, Inc. (CST), Danvers, MA, USA] and GAPDH (1:5,000; cat. no. AF1186/AF0006; Beyotime Institute of Biotechnology) at $4^{\circ} \mathrm{C}$ overnight. Following washing with TBS three times, the membranes were incubated with goat anti-rabbit (1:5,000; cat. no. D111018) or goat anti-mouse (1:5,000; cat. no. D110099) immunoglobulin G-horseradish peroxidase (BBI Life Sciences, Shanghai, China) antibodies at $37^{\circ} \mathrm{C}$ for 2 h. Luminescent liquid (cat. no. KLS0500; EMD Millipore, Billerica, MA, USA) was added for color rendering. The film was scanned and the net optical density value of the strip was analyzed using a gel image processing system (Image-Pro Plus v7.0; Media Cybernetics, Inc. Rockville, MD, USA). The relative expression of the target protein was divided by that of the internal control.

ELISA analysis. The concentration of CX3CL1 in the blood was detected using Fractalkine ELISA kits (cat. no. DCX310; R\&D Systems, Inc.), in accordance with the manufacturer's protocol.

Reverse transcription-quantitative polymerase chain reaction (RT-qPCR) analysis. Total RNA from prostate carcinoma tissues, healthy vertebrae tissues, healthy limb bone tissues and prostate cancer cells was extracted using TRIzol ${ }^{\circledR}$ (Invitrogen; Thermo Fisher Scientific, Inc.), according to the manufacturer's protocol. A total of $2 \mu \mathrm{g}$ RNA was reverse transcribed $\left(65^{\circ} \mathrm{C}\right.$ for $5 \mathrm{~min}, 37^{\circ} \mathrm{C}$ for $15 \mathrm{~min}$ and $98^{\circ} \mathrm{C}$ for $5 \mathrm{~min}$ ) to obtain cDNA using a reagent kit (Invitrogen; Thermo Fisher Scientific, Inc.). Subsequently, qPCR analyses were conducted using the QuantiNova $^{\mathrm{TM}}$ SYBR ${ }^{\circledR}$ Green PCR kit (Qiagen GmbH, Hilden, Germany) and the qPCR data collection was performed using a thermocycler (ABI 7500; Thermo Fisher Scientific, Inc.). PCR conditions were as follows: $95^{\circ} \mathrm{C}$ for $2 \mathrm{~min}, 94^{\circ} \mathrm{C}$ for $20 \mathrm{sec}$, $58^{\circ} \mathrm{C}$ for $20 \mathrm{sec}$ and $72^{\circ} \mathrm{C}$ for $20 \mathrm{sec} ; 40$ cycles. The expression ratio was calculated according to the $2^{-\Delta \Delta \mathrm{Cq}}$ method (22) and data were normalized to GAPDH. The primer sequences are presented in Table I.

Immunohistochemical and immunofluorescence staining. Tissue specimens fixed in $4 \%$ polyoxymethylene (cat. no. P1110; Beijing Solarbio Science \& Technology Co., Ltd.; $>24 \mathrm{~h}$ at room temperature) and embedded in paraffin were sectioned to $4 \mu \mathrm{m}$ thick. The sections were sequentially placed in xylene I for $15 \mathrm{~min}$, xylene II for $15 \mathrm{~min}$, absolute ethanol I for $5 \mathrm{~min}$, absolute ethanol II for $5 \mathrm{~min}, 85 \%$ alcohol for $5 \mathrm{~min}$, $75 \%$ alcohol for $5 \mathrm{~min}$ and a distilled water wash. Antigenic retrieval was performed using sodium citrate $(\mathrm{pH}$ 6.0; cat. no. G1202; Wuhan Servicebio Technology Co., Ltd., Wuhan, China); samples were placed for $8 \mathrm{~min}$ in a microwave oven to boil, followed by $8 \mathrm{~min}$ with the microwave turned off for heat preservation, followed by a medium heat. The sections were incubated in $\mathrm{H}_{2} \mathrm{O}_{2}$ (3\%) for $10 \mathrm{~min}$, and blocked in 5\% goat serum (cat. no. AR1010; Wuhan Boster Biological Technology, Ltd., Wuhan, China) for $60 \mathrm{~min}$ at room temperature, followed by anti-CX3CL1 or anti-CX3CR1 or androgen receptor (AR; 1:500; cat. no. 5153; CST) antibodies at $37^{\circ} \mathrm{C}$ for $60 \mathrm{~min}$. Following incubation with the secondary antibody for $45 \mathrm{~min}$ at room temperature, specimens were stained with 3,3'-diaminobenzidine buffer (1:11; cat. no. K5007; Dako; Agilent Technologies, Inc., Santa Clara, CA, USA) for $1 \mathrm{~min}$, followed by tap water flushing to stop the staining at room temperature. The sections were all counterstained with hematoxylin for 3 min, dehydrated and mounted in neutral balsam (cat. no. G8590; Beijing Solarbio Science \& Technology Co., Ltd.). Observation was performed used a microscope (ECLIPSE TI-SR; Nikon Corporation, Tokyo, Japan) at x100 magnification. Immunofluorescence was performed following a procedure described previously (23). Immunofluorescence was conducted to detect the expression of F-actin (1:100; cat. no. ab205; Abcam) in cells. Observation was performed at x400 magnification.

Xenograft study. A total of 18 male NOD/SCID mice at 4-6 weeks of age (14-20 g) were obtained from the Vital River Laboratory Animal Technology Co., Ltd. (Beijing, China). The animal feeding environment was as follows: $22-27^{\circ} \mathrm{C}$; humidity, 40-70\%; light/dark cycle, 12 ; free access to sterilized feed and water. The animal studies were approved by the Animal Ethics Committee of Zhongshan Hospital, Fudan University. These mice were randomly divided into two equal groups. A total of $\sim 1 \times 10^{6} \mathrm{CX} 3 \mathrm{CR} 1$-overexpressing PC- 3 cells or control cells were suspended in $200 \mu 1$ serum-free medium 
and injected into the left ventricle of the mice following sodium pentobarbital anesthesia, as described previously (24). After 6-8 weeks, the mice received a positron emission tomography (PET) scan for fluorodeoxyglucose localization. If a suspected spinal metastasis was found, the lesion underwent a further micro-computed tomography (CT) scan and pathological examination.

Statistical analyses. The data are expressed as the mean \pm standard deviation. The analyses were performed using SPSS statistical software version 16.0 (SPSS, Inc., Chicago, IL, USA). The $\chi^{2}$ test was used for the xenograft study (spinal tumorigenesis rate). Student's t-tests and one-way analysis of variance followed by Tukey's post-hoc test were used to make statistical comparisons between groups (clinical samples and cell samples). All experiments were repeated at least three times. $\mathrm{P}<0.05$ was considered to indicate statistical significance.

\section{Results}

CX3CL1/CX3CR1 is overexpressed in human prostate cancer tissue and cell lines. To investigate the potential role of CX3CL1/CX3CR1 in prostate cancer spinal metastasis, 12 cases of prostate carcinoma with spinal metastasis, 12 cases of primary spinal tumor, 12 samples of healthy vertebrae and 12 samples of healthy limb bone tissues (fractures) were collected for western blot and RT-qPCR analyses to measure the expression of CX3CL1/CX3CR1 (Figs. 1 and 2). The results demonstrated that the expression of CX3CL1/CX3CR1 in metastatic spinal lesions from prostate cancer was higher compared with primary spinal tumors $(\mathrm{P}<0.01$ and $\mathrm{P}<0.05$; Figs. 1A, B and 2A). Additionally, CX3CL1 was highly expressed in healthy spinal osseous tissues compared with healthy limb osseous tissues $(\mathrm{P}<0.01$ and $\mathrm{P}<0.05$; Figs. 1A, B and 2B). The ELISA analysis demonstrated that the average concentration of CX3CL1 in the blood was $0.31 \mathrm{ng} / \mathrm{ml}$ in prostate cancer tissues with spinal metastasis, which was increased compared with the 0.12 and $0.16 \mathrm{ng} / \mathrm{ml}$ observed in primary tumors and healthy human blood, respectively $(\mathrm{P}<0.05$; Fig. 1C). Furthermore, it was identified that the protein expression of CX3CR1 was significantly increased in prostate carcinomas with spinal metastasis compared with primary spinal tumors ( $\mathrm{P}<0.05$; Figs. 1D, E and 2C).

In human prostate cancer cells (VCaP, PC-3, LNCap and 22RV1) and prostate epithelial cells (RWEP-1), it was observed that the expression of CX3CR1 was higher in prostate cancer cells compared with normal prostate epithelial cells, as indicated by western blotting and RT-qPCR (Fig. 1F-H). Furthermore, the expression of CX3CL1 and CX3CR1 was detected in prostate carcinomas with spinal metastasis and primary tumor tissues by immunohistochemistry, and it was revealed that the expression of CX3CL1 and CX3CR1 was increased in prostate carcinomas with spinal metastasis (Fig. 1I and J). Similar results were observed in healthy spines and healthy limbs. It was additionally demonstrated that the expression of CX3CR1 was increased in prostate cancer tissues compared with healthy prostate tissues (Fig. 1J).

CX3CR1 promotes cell proliferation and inhibits cellular apoptosis. To examine the potential role of CX3CR1 in prostate
Table I. Primer sequences for CX3CL1/CX3CR1.

\begin{tabular}{lc}
\hline Name & \multicolumn{1}{c}{ Sequence $\left(5^{\prime} \rightarrow 3^{\prime}\right)$} \\
\hline CX3CL1 & F: 5'-GACCCCTAAGGCTGAGGAAC-3' \\
CX3CL1 & R: 5'-AGAAGAGGAGGCCAAGGAAG-3' \\
CX3CR1 & F: 5'-GACGGTTGCATTTAGCCATT-3' \\
CX3CR1 & R: 5'-TGCTCAGAACACTTCCATGC-3' \\
GAPDH & F: 5'-GCGAGATCCCTCCAAAATCAA-3' \\
GAPDH & R: 5'-GTTCACACCCATGACGAACAT-3'
\end{tabular}

CX3CL1, C-X3-C motif chemokine ligand 1; CX3CR1, C-X3-C motif chemokine receptor $1 ; \mathrm{F}$, forward; $\mathrm{R}$, reverse.

cancer cell function, a lentivirus was used to construct stable $\mathrm{OE}$ and NC cell lines, in VCaP and PC-3 cells. The cells were transfected with either the specific siRNA or NC to suppress the expression of CX3CR1. The expression of CX3CR1 protein and mRNA is presented in Fig. 3A and B. Subsequently, the cell proliferation of these cells was measured by CCK-8 assays. As exhibited in Fig. 3C and D, overexpression of CX3CR1 promoted cell proliferation and knockdown of CX3CR1 reduced cell proliferation. In addition, cellular apoptosis was measured by flow cytometry, and it was observed that the overexpression of CX3CR1 inhibited cellular apoptosis, and the repression of CX3CR1 increased cellular apoptosis (Fig. 3E and F). These results suggested that $\mathrm{CX} 3 \mathrm{CR} 1$ expression may be associated with prostate cancer cell proliferation and apoptosis.

CX3CL1 induces cell migration and invasion. The effects of CX3CL1/CX3CR1 on cell migration and invasion were further detected by scratch wound and Transwell assays. The scratch wound assays revealed that treatment with CX3CL1 (100 nM) or overexpression of CX3CR1 in VCaP cells increased the cell migration rates $(\mathrm{P}<0.05)$, and knockdown of CX3CR1 decreased $\mathrm{VCaP}$ cell migration rates $(\mathrm{P}<0.01)$, as presented in Fig. 4A-C. Additionally, cell invasion was assessed by Transwell assay, and it was observed that PC-3 cell invasion was regulated by CX3CR1 expression. In CX3CL1 (100 nM)-treated or CX3CR1-overexpressing PC-3 cells, cell invasion was increased by 25.95 and $43.63 \%$, respectively (Fig. 4D and E). However, in CX3CR1-knockdown PC-3 cells, cell invasion decreased by $38.89 \% \quad(\mathrm{P}<0.05$; Fig. 4F). Additionally, the present study aimed to determine whether the organization of the actin cytoskeleton was altered in CX3CR1-overexpressing or knockdown cells. As presented in Fig. 4G, in CX3CL1-treated cells or CX3CR1overexpressing cells, F-actin structures formed lamellipodial protrusions around the periphery of the cells. However, in CX3CR1-knockdown PC-3 cells, the actin-rich membrane ruffles were absent. The results of the present study suggested that CX3CL1 may serve important roles in prostate cancer cell migration and invasion.

CX3CL1/CX3CR1 activates the SrC/FAK pathway. Previous work demonstrated that CX3CL1 is associated with spinal metastasis in different types of cancer (17) and that the Src/FAK signaling pathway is associated with spinal metastasis 
A Paracancerous tissues Healthy tissues
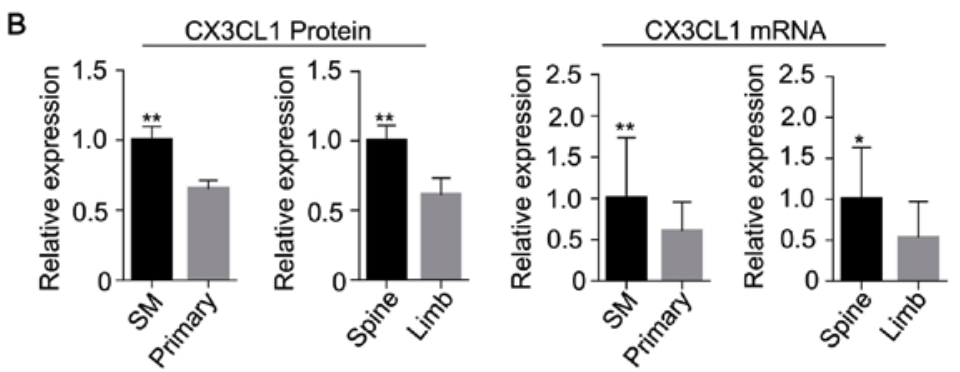

C

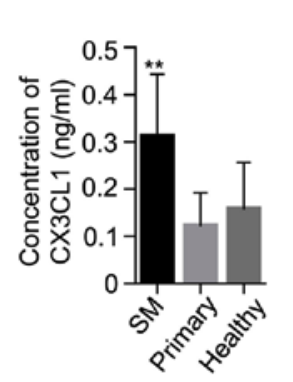

D
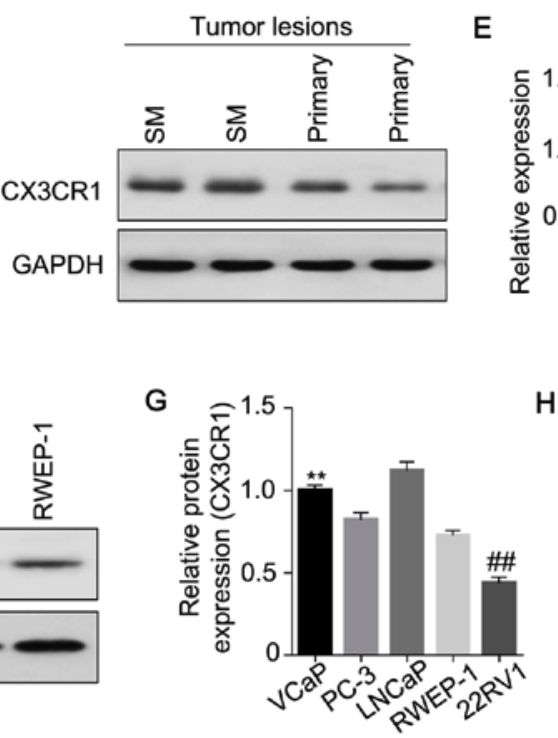

Protein

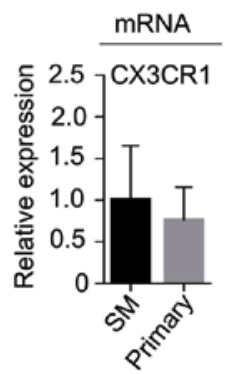

$\mathbf{F}$

G

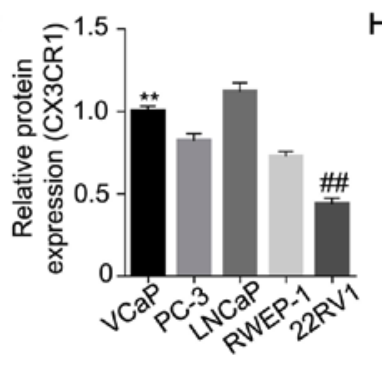

$\mathrm{H}$
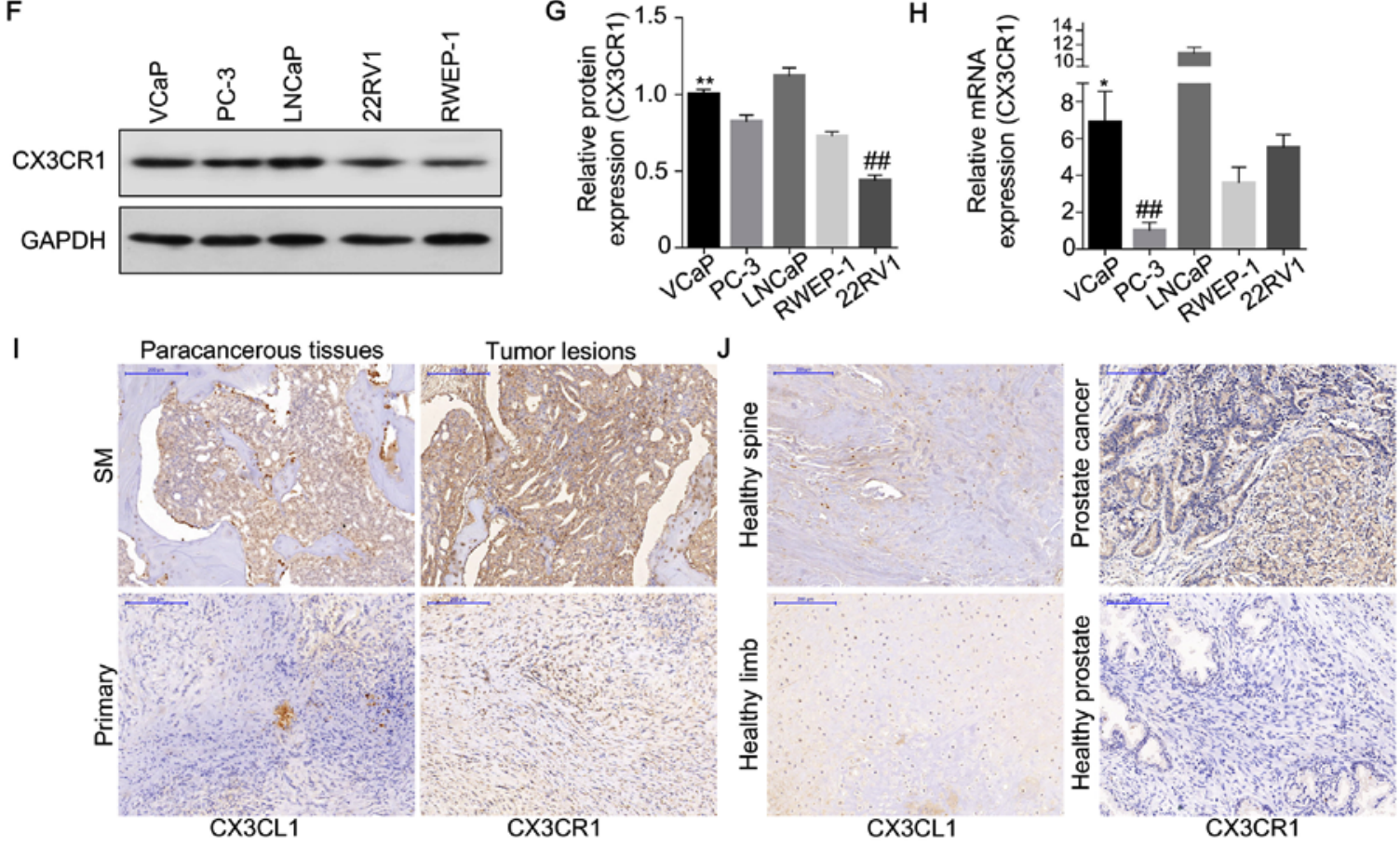

Figure 1. CX3CL1/CX3CR1 is overexpressed in human prostate cancer tissues and cell lines. CX3CL1 was overexpressed in the paracancerous tissues of spinal metastasis (SM) and healthy spines, as demonstrated by (A) western blotting, (B) densitometric analysis of western blotting, and RT-qPCR of the CX3CL1 mRNA expression level; GAPDH was as a loading control. ${ }^{*} \mathrm{P}<0.05,{ }^{* *} \mathrm{P}<0.01$ vs. respective control. $\mathrm{n}=12$. (C) The concentration of $\mathrm{CX} 3 \mathrm{CL} 1$ in the blood of patients with prostate cancer with SM, primary tumors and healthy cases was measured by ELISA. ${ }^{* *} \mathrm{P}<0.01$ vs. primary; ${ }^{\# \#} \mathrm{P}<0.01$ vs. healthy. CX3CR1 was overexpressed in prostate cancer with SM compared with primary cancer tissues as demonstrated by (D) western blotting, (E) densitometric analysis of western blotting, and RT-qPCR of the CX3CR1 mRNA expression level; GAPDH was used as a loading control. "P<0.05 vs. respective control. $\mathrm{n}=12$. CX3CR1 expression in VCaP cells (derived from SM) was increased compared with 22RV1 cells (derived from primary tumors) and RWEP-1 cells (healthy prostate epithelial cells), as demonstrated by (F) western blotting and (G) densitometric analysis. ${ }^{* *} \mathrm{P}<0.01$ vs. $22 \mathrm{RV} 1$; $^{\# \#} \mathrm{P}<0.01 \mathrm{vs}$. RWEP1. (H) The mRNA expression of CX3CR1 in VCaP cells was increased compared with $22 \mathrm{RV} 1$ and PC-3 cells. ${ }^{*} \mathrm{P}<0.05$ vs. $22 \mathrm{RV} 1$; $^{\# \#} \mathrm{P}<0.01 \mathrm{vs}$. VCaP. (I) Clinical samples were collected and examined by immunohistochemical staining with CX3CL1 and CX3CR1 antibodies. Representative staining images are presented (x100 magnification). (J) Immunohistochemical staining of CX3CL1 and CX3CR1 is presented in healthy human spinal tissue, limb osseous tissues, primary prostate cancer and healthy prostate tissues (x100 magnification). SM, spinal metastasis; RT-qPCR, reverse transcription-quantitative polymerase chain reaction; CX3CL1, C-X3-C motif chemokine ligand 1; CX3CR1, C-X3-C motif chemokine receptor 1.

(data not shown). The IPA database predicted that kinases, including Src, protein-tyrosine kinase 2- $\beta$ (PTK2B) and FAK were associated with $\mathrm{CX} 3 \mathrm{CL} 1 / \mathrm{CX} 3 \mathrm{CR} 1$ in prostate cancer cells (Fig. 5). Therefore, the activities of these kinases were examined by detecting their phosphorylated expression levels. Following treatment with CX3CL1 $(100 \mathrm{nM})$, the expression of phosphorylated Src (Tyr416) and phosphorylated FAK (Tyr576/577) increased in a time-dependent manner (Fig. 6A). In addition, the phosphorylation of epidermal growth factor receptor (EGFR) (Tyr992) was markedly induced (Fig. 6B). However, the phosphorylated PTK2B did not alter following treatment with CX3CL1 (Fig. 6B). Additionally, 

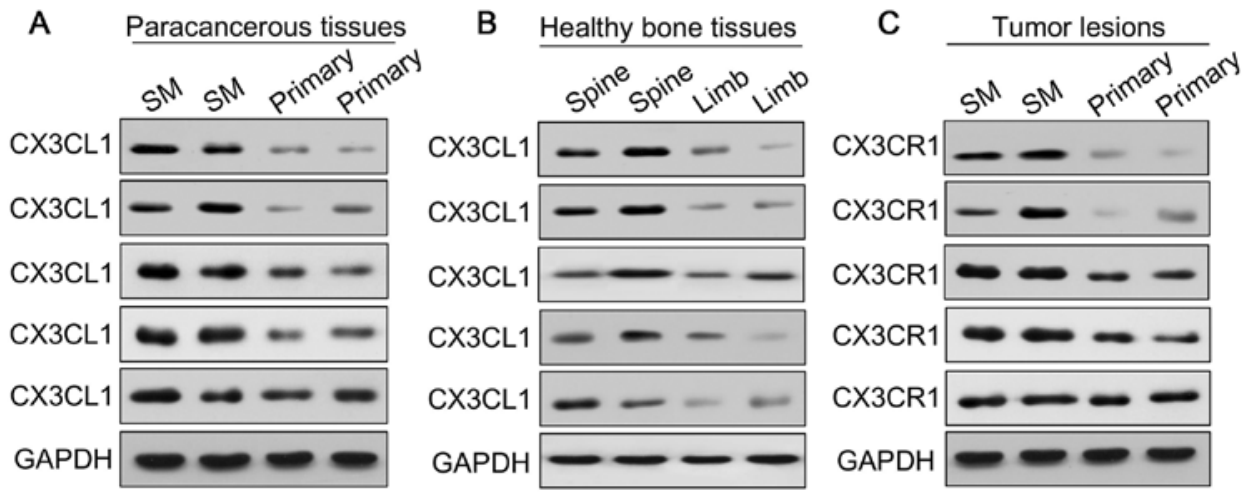

Figure 2. Expression of CX3CL1/CX3CR1 in human tissues. Clinical samples of (A) paracancerous tissues, (B) healthy bone tissues and (C) tumor tissues were collected and examined by western blotting using CX3CL1 and CX3CR1 antibodies. GAPDH was used as a loading control. CX3CL1, C-X3-C motif chemokine ligand 1; CX3CR1, C-X3-C motif chemokine receptor 1.

CX3CR1-overexpressing and knockdown cells were used to detect the phosphorylation of EGFR, FAK and Src. The results revealed that similar to treatment with CX3CL1, overexpression of CX3CR1 increased the phosphorylation of EGFR, FAK and Src, while downregulating CX3CR1 decreased the phosphorylation of EGFR, FAK and Src in VCaP cells (Fig. 6C and D).

Subsequently, the Src inhibitor bosutinib, the FAK inhibitor PF-562271, and the EGFR inhibitor afatinib were used to further investigate the signaling pathway involved in CX3CL1/CX3CR1-induced prostate cancer progression. The results demonstrated that CX3CL1-induced Src and FAK phosphorylation were blocked by their corresponding inhibitors (Fig. 6E and F). Furthermore, the EGFR inhibitor afatinib markedly reduced the phosphorylation of Src (Fig. 6G); however, the Src inhibitor bosutinib did not inhibit the phosphorylation of EGFR (Fig. $6 \mathrm{H}$ ), suggesting that $\mathrm{Src}$ is a downstream effector of EGFR in a CX3CL1/CX3CR1-associated pathway. Furthermore, the expression of MMP-9 and ROCK1 was regulated by CX3CR1 expression (Fig. 6I and J). Functionally, the inhibitors of FAK, Src and EGFR reversed the induction of cell migration caused by treatment with CX3CL1, as evaluated by scratch wound assay (Fig. 7).

CX3CL1/CX3CR1 facilitates spinal metastasis of prostate cancer in vivo. Finally, the present study employed xenograft mouse models to verify the association between CX3CL1 and spinal metastasis in prostate cancer. PC3 cells were injected into the left ventricles of the mice, which was the closest way to mimic the clinical metastasis of the tumor. It was observed that four out of nine (44.44\%) and one out of nine $(11.11 \%)$ mice in the CX3CR1-overexpressing and control cell groups, respectively, formed metastases. All the metastases in the CX3CR1-overexpressing cell injected group involved the spine, while the control cell injected group exhibited metastasis to the maxillofacial bones (Fig. 8). PET scans demonstrated that fluorodeoxyglucose was concentrated in the locality of the spine. Furthermore, micro-CT scans revealed that lesions in the affected vertebrae, indicating that they were damaged in CX3CR1-overexpressing cell-injected mice (Fig. 9A). The diagnosis of spinal metastasis of prostate cancer was verified by hematoxylin and eosin staining and positive immunohistochemical staining of AR (Fig. 9B). It was additionally identified that one case in the CX3CR1 overexpression group exhibited femoral metastases accompanied by spinal metastases. In addition, CX3CR1 exhibited increased expression in prostate cancer tissues compared with healthy spines (Fig. 9B and C). The results of the present study demonstrated that CX3CL1/CX3CR1 induced spinal metastasis in an in vivo model.

\section{Discussion}

The present study reported that the expression of CX3CL1/CX3CR1 in prostate cancer tissues with spinal metastasis was increased compared with primary tumors, and the expression of CX3CL1 in healthy spines was increased compared with healthy limb osseous tissues. In addition, in prostate cancer cell lines, the expression of CX3CR1 was increased in prostate cancer cells lines that were derived from metastases of the spine, bone and lymph nodes when compared with cells that were derived from primary tumors, and healthy human prostate epithelial cells. Furthermore, overexpression of CX3CL1/CX3CR1 induced cell proliferation, migration and invasion, while downregulation of CX3CL1/CX3CR1 reduced cell proliferation, migration and invasion and induced cellular apoptosis. It was also observed that CX3CL1/CX3CR1 increased the phosphorylation of EGFR, FAK and Src. The inhibitors of these kinases reversed the effects of treatment with CX3CL1 or overexpression of CX3CR1, and inhibited phosphorylation of these kinases and cell migration, suggesting that the EGFR, FAK and Src signaling pathways may be involved in CX3CL1/CX3CR1-induced prostate cancer progression. Furthermore, overexpression of CX3CR1 induced spinal metastasis in an in vivo prostate cancer model. Therefore, the present study demonstrated for the first time, to the best of our knowledge, that CX3CL1/CX3CR1 promotes prostate cancer spinal metastasis by regulating the Src/FAK pathway, suggesting that CX3CL1/CX3CR1 may be a potential target in future studies aimed at preventing prostate cancer progression.

Chemokines serve important roles in the progression of cancer. Previous studies have demonstrated that the CX3CL1/CX3CR1 axis is involved in the proliferation, survival and metastasis of various malignant tumor types (11-13). In prostate cancer, the CX3CL1/CX3CR1 axis activates the phosphatidylinositol 3-kinase/RAC- $\alpha$ serine/threonine-protein 

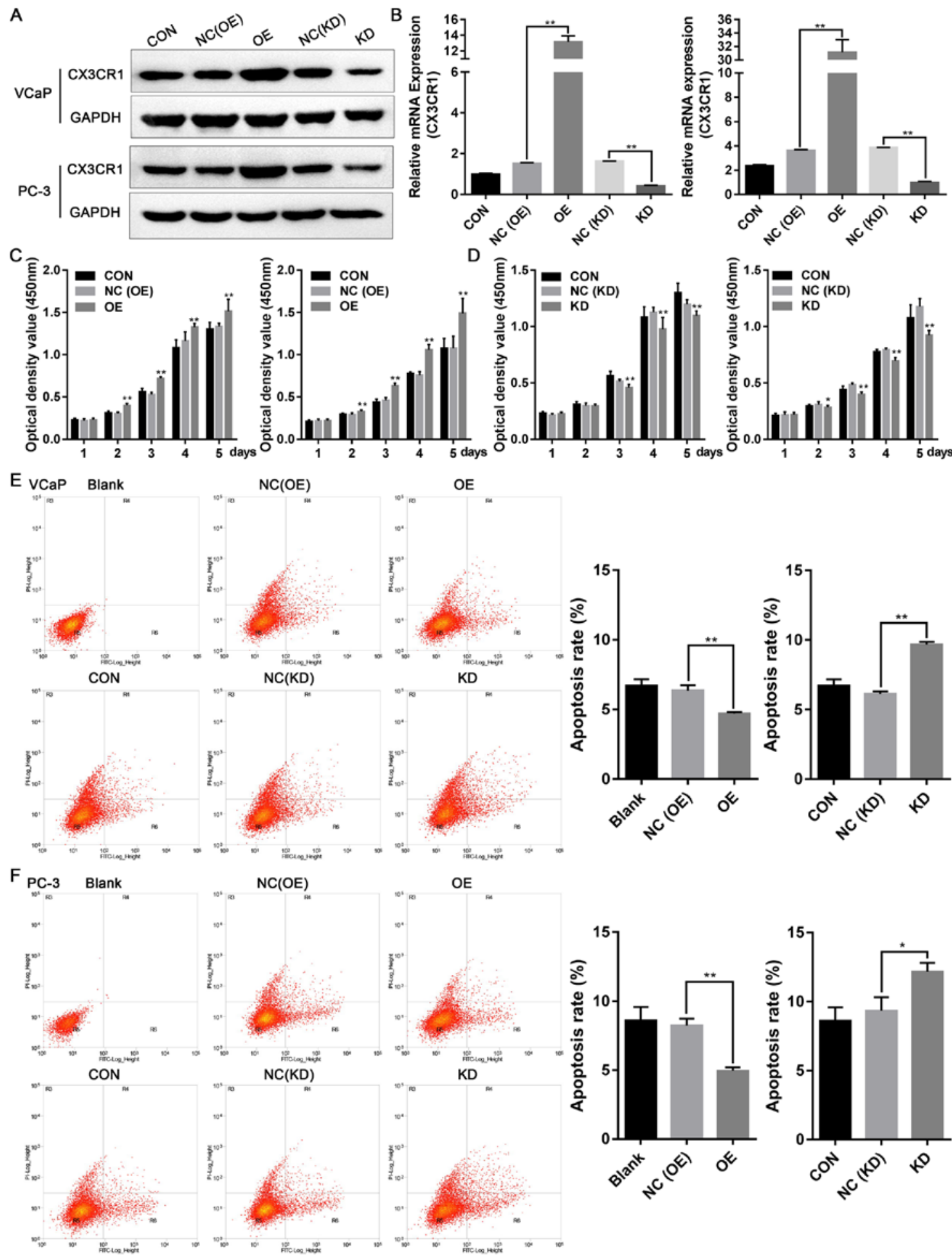

Figure 3. CX3CR1 promotes cell proliferation and inhibits cellular apoptosis. Stably-overexpressing CX3CR1 and control cell lines (VCaP and PC-3) were constructed by lentivirus infection. Specific siRNA was used to inhibit the expression of CX3CR1 (KD) in VCaP and PC-3 cells. The expression of CX3CR1 was determined by (A) western blotting and reverse transcription-quantitative polymerase chain reaction analysis (B, left: VCaP; right: PC-3). Cell counting kit-8 assays was used to demonstrate VCaP and PC-3 cell proliferation following lentivirus infection (C, left: VCaP; right: PC-3) or specific siRNA transfection (D, left: VCaP; right: PC-3). ${ }^{*} \mathrm{P}<0.05,{ }^{* *} \mathrm{P}<0.01$ vs. respective NC group. The experiment was repeated three times. Annexin V-FITC/PI assays were used to detect the cellular apoptosis of (E) VCaP and (F) PC-3 cells following lentivirus infection or siRNA transfection. The experiment was repeated three times. ${ }^{*} \mathrm{P}<0.05,{ }^{* *} \mathrm{P}<0.01$. OE, overexpression; NC, negative control; KD, knockdown; CON, control; CX3CL1, C-X3-C motif chemokine ligand 1; CX3CR1, C-X3-C motif chemokine receptor 1; siRNA, small interfering RNA; FITC, fluorescein isothiocyanate; PI, propidium iodide.

kinase pathway and serves an essential role in skeletal metastasis (16). Additionally, CX3CL1/CX3CR1 increases invasion and metastasis by promoting epithelial-to-mesenchymal transition through disintegrin and metalloproteinase domain-containing protein 17/protransforming growth factor- $\alpha$ /EGFR pathway in hypoxic androgen-independent prostate cancer cells (13). A recent study using microarray analysis reported that patients with spinal metastases 
A

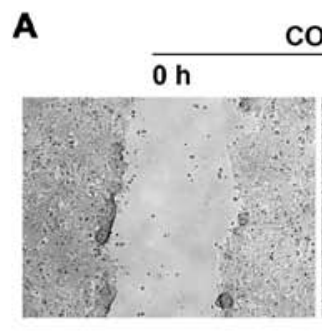

B
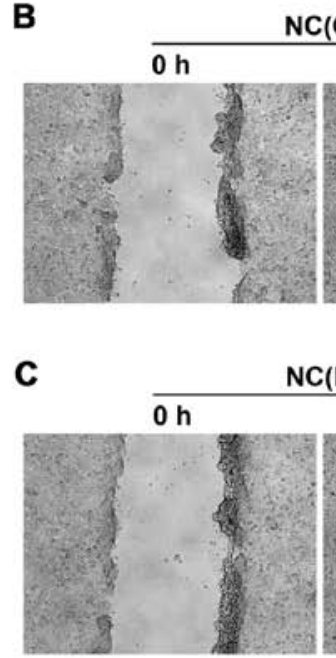

D $\mathrm{CON}$
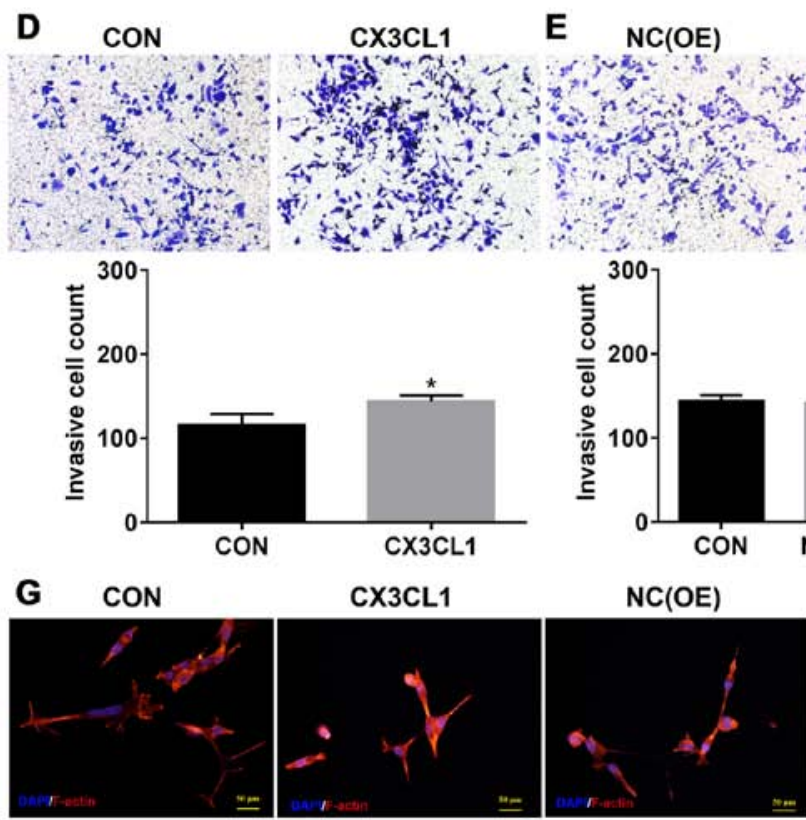

E $\mathrm{NC}(\mathrm{OE})$

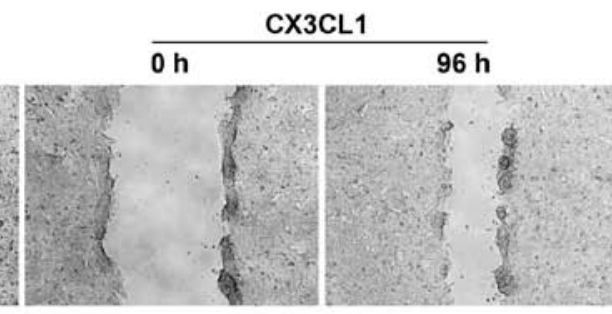

CX3CL1

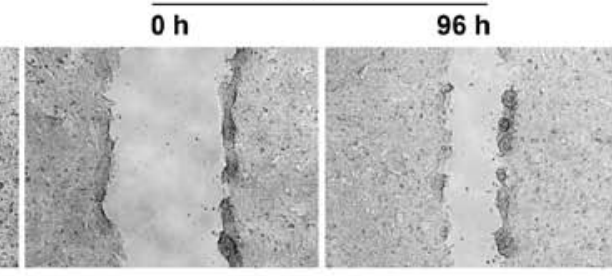

sin
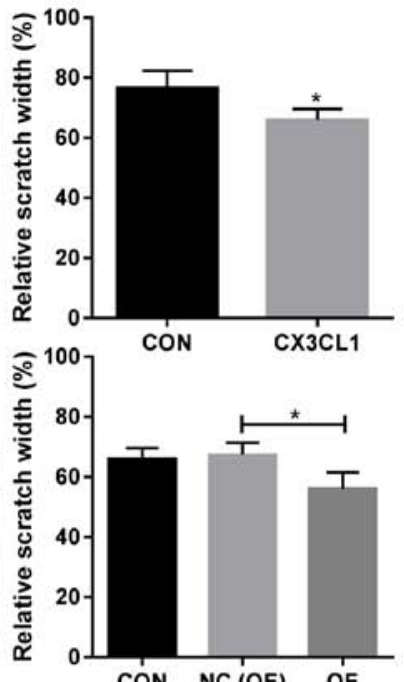

CON NC (OE) OE

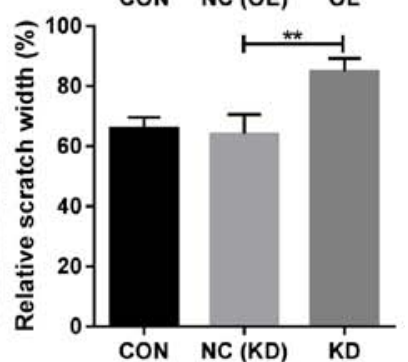

F $\quad \mathrm{NC}(\mathrm{KD})$

KD
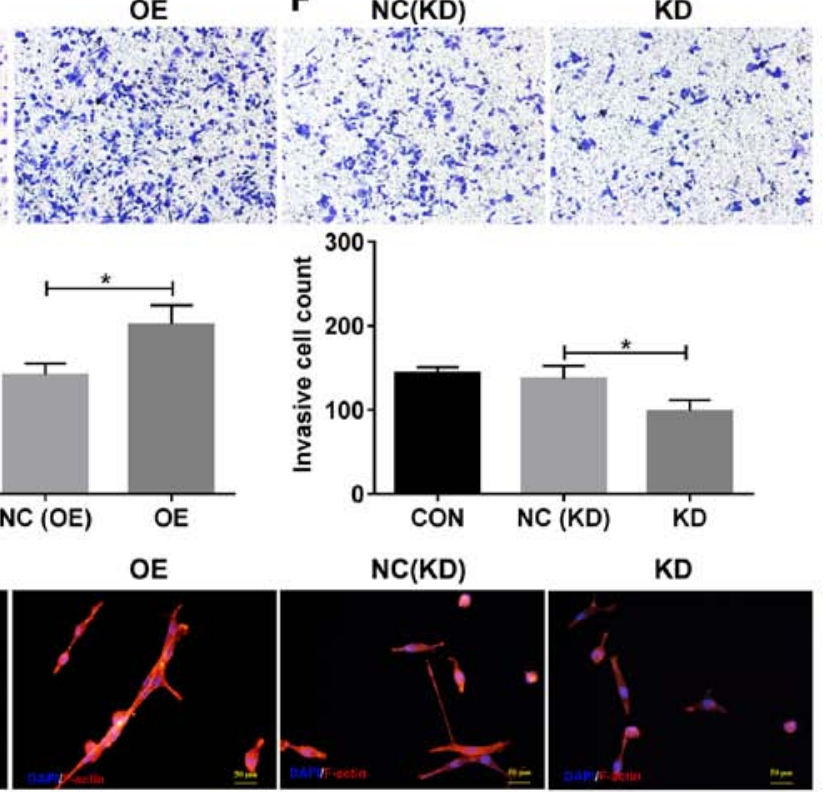

Figure 4. CX3CL1 induces cell migration and invasion. Cell migration was measured by scratch wound assay in (A) CX3CL1-treated, (B) CX3CR1 overexpression and (C) CX3CR1-knockdown group at time point of $96 \mathrm{~h}$. Representative images are presented (left panel; x100 magnification). The results were summarized from three independent experiments (right panel). ${ }^{*} \mathrm{P}<0.05,{ }^{* *} \mathrm{P}<0.01$ vs. respective control. Cell invasion was determined by Transwell assay in (D) CX3CL1-treated, (E) CX3CR1 overexpression and (F) CX3CR1-knockdown group. Representative images are presented (upper panel; x200 magnification). The results were summarized from three independent experiments (lower panel). $\mathrm{P}<0.05$ vs. respective control. (G) Cells were fixed and stained with F-actin in CX3CL1 treated, CX3CR1 overexpression and CX3CR1 knockdown group, the representative images are shown (magnification, $\mathrm{x} 400$ ). OE, overexpression; NC, negative control; KD, knockdown; CON, control; CX3CL1, C-X3-C motif chemokine ligand 1.

from prostate cancer exhibited significantly high levels of CX3CL1 (17). The results of the present study demonstrated that CX3CL1 was highly expressed in healthy spinal osseous tissues compared with healthy limb osseous tissues, suggesting that prostate cancer was more prone to metastasis to spinal osseous tissues compared with limb osseous tissues. Additionally, in prostate cancer tissues with spine metastasis, the expression of CX3CL1/CX3CR1 was increased compared with primary prostate cancer, suggesting that $\mathrm{CX} 3 \mathrm{CL} 1 / \mathrm{CX} 3 \mathrm{CR} 1$ promotes the spinal metastasis of prostate cancer. As the CX3CL1/CX3CR1 axis was involved in the progression of different types of tumor, the present results suggested that CX3CL1/CX3CR1 may serve essential roles in spine metastasis in other types of tumor in 


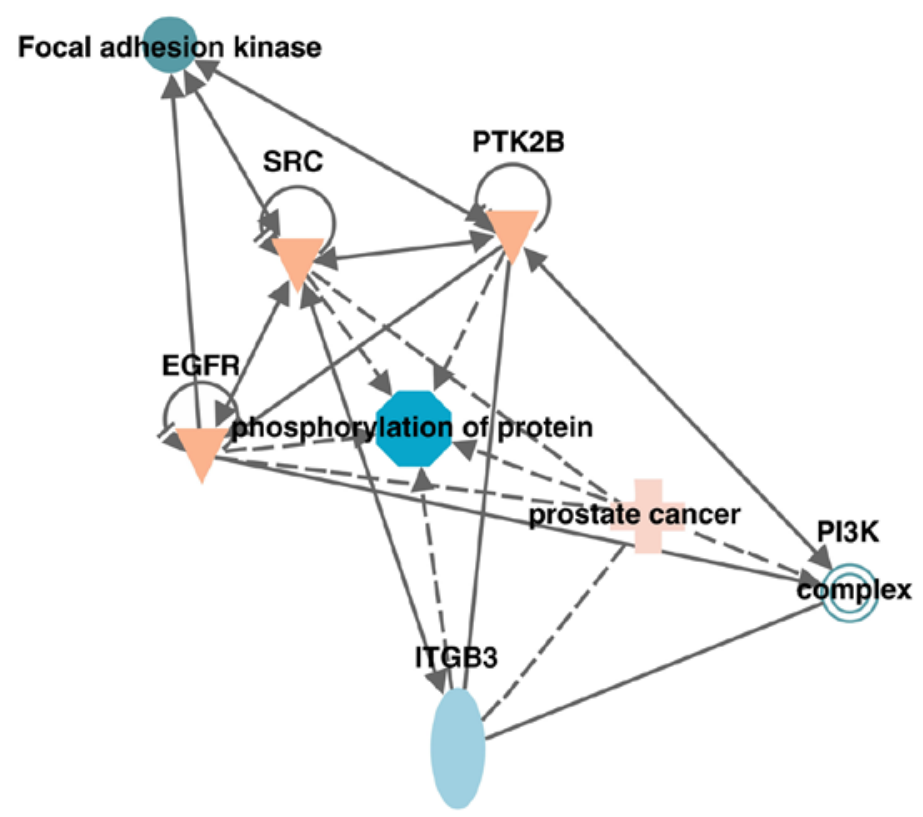

Figure 5. Kinases associated with the CX3CL1/CX3CR1 axis. The association between kinases relevant to CX3CL1 in the Src/focal adhesion kinase signaling pathway were predicted using the Ingenuity Pathway Analysis database. Src, proto-oncogene tyrosine-protein kinase Src; PI3K, phosphatidylinositol 3-kinase; ITGB3, integrin $\beta-3$; EGFR, epidermal growth factor receptor; PTK2B, protein-tyrosine kinase 2- $\beta$.

addition to prostate cancer. The present data demonstrated that overexpression of CX3CR1 induced cell proliferation, migration and invasion, while downregulation of CX3CR1 reduced these processes and increased cellular apoptosis. Recently, a number of studies reported that CX3CR1 induced apoptosis resistance in different types of cancer (25-27). The present results were confirmed by these studies, suggesting that knockdown of CX3CR1 may serve a suppressive role in tumors, and CX3CL1/CX3CR1 may serve as a therapeutic target in prostate cancer. VCaP cells were established from a vertebral metastatic lesion with lower metastatic potential compared with PC-3 cells; PC-3 cells were selected to detect the cell motility, and it was observed that CX3CL1/CX3CR1 promoted prostate cancer cell migration, invasion and actin organization, while suppression of CX3CR1 inhibited cell migration and invasion as demonstrated by scratch wound assay and Transwell assay, consistent with a previous study (28). As ROCK1 is a key molecule in the regulation of the cytoskeleton $(29,30)$, the present study detected the expression of ROCK1 and demonstrated that CX3CL1/CX3CR1 regulated ROCK1 expression. These results suggest that CX3CL1/CX3CR1 increases cell migration rates by regulating cytoskeleton formation.

Src/FAK signaling is known for its important effects on cell migration through the reorganization of the cytoskeleton (31). The activation of Src is achieved by the sequential phosphorylation/autophosphorylation of tyrosine residues (Tyr527 and Tyr416) (32). FAK is a widely expressed cytoplasmic protein-tyrosine kinase involved in cell spreading, migration and survival (33-35). Recruitment of the Src family kinases leads to the phosphorylation of Tyr576 and Tyr577 in the catalytic domain of FAK (36). In addition, numerous studies have demonstrated that aberrant activation of the Src family of kinases serves important roles in the bone metastasis of prostate cancer (37). To explain the effects of
CX3CL1/CX3CR1 on cell migration, the phosphorylation of Src and FAK was detected, and it was observed that treatment with CX3CL1 or overexpression of CX3CR1 increased the expression of phosphorylated Src and FAK. Functionally, the inhibitors of EGFR, Src or FAK reversed cell migration induced by CX3CL1/CX3CR1, suggesting that the Src/FAK pathway is involved in CX3CL1/CX3CR1-induced cell migration. Furthermore, we observed that the expression of MMP-9 was increased in CX3CR1 overexpressing cells, which may explain the role of CX3CL/CX3CR1 in cell invasion.

The predicted results from the IPA database demonstrated that EGFR and PTK2B interact with Src. The present data demonstrated that the phosphorylation of EGFR was increased following treatment with CX3CL1 or overexpression of CX3CR1. In addition, the EGFR inhibitor afatinib inhibited the phosphorylation of Src, while the inhibitor of Src did not block the phosphorylation of EGFR, verifying that $\mathrm{Src}$ is a downstream effector of EGFR in a CX3CL1-associated pathway. These results revealed that the Src/FAK signaling pathway is activated by CX3CL1 and the phosphorylation of EGFR is required for Src/FAK activation in prostate cancer cells. The PC-3 cell line was established from a bone metastasis of a patient with grade IV prostate cancer and is frequently used as a model of subcutaneous tumors in mice. Therefore, the PC3 cell line was used to detect the effects of CX3CL1/CX3CR1 on prostate cancer progression in an in vivo model, and demonstrated that the final number of spinal metastasis cases increased in the CX3CR1-overexpressing group compared with the control group, which is consistent with the clinical specimen pathological findings. These data further demonstrated that CX3CL1/CX3CR1 induces spinal metastasis in prostate cancer and serves important roles in prostate cancer progression.

In conclusion, the CX3CL1/CX3CR1 axis promoted prostate cancer cell proliferation, migration and invasion through the Src/FAK pathway in vitro. Future studies are required 
A

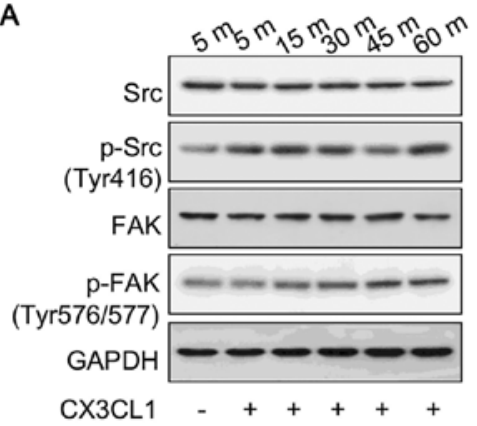

C

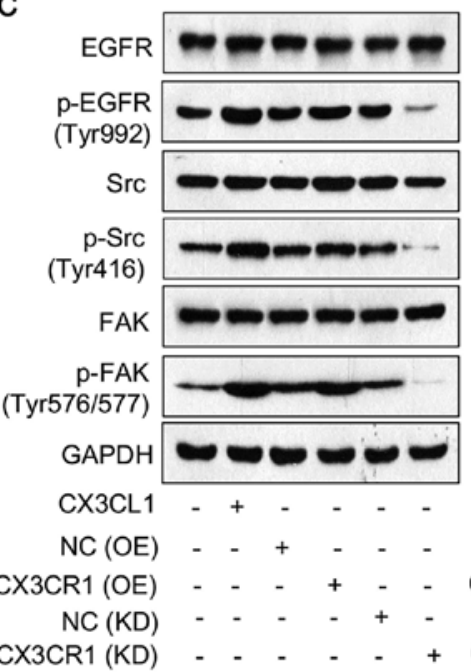

B

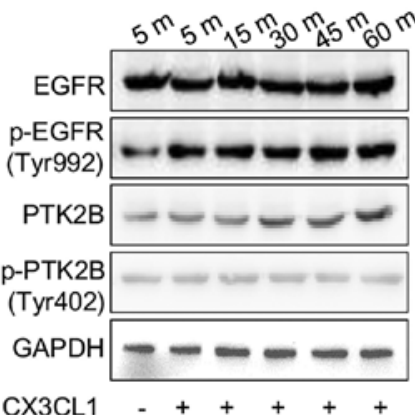

D

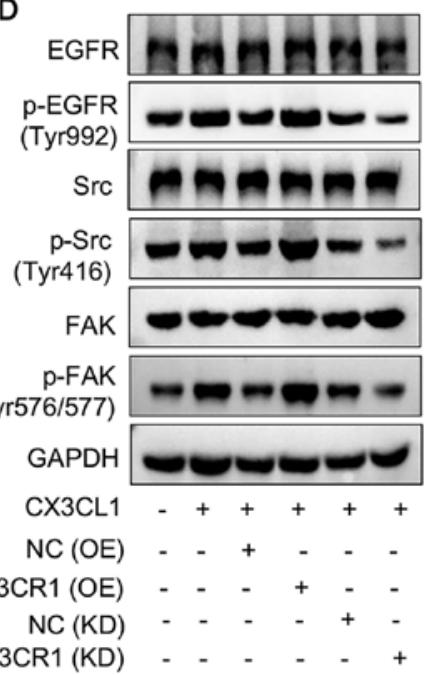

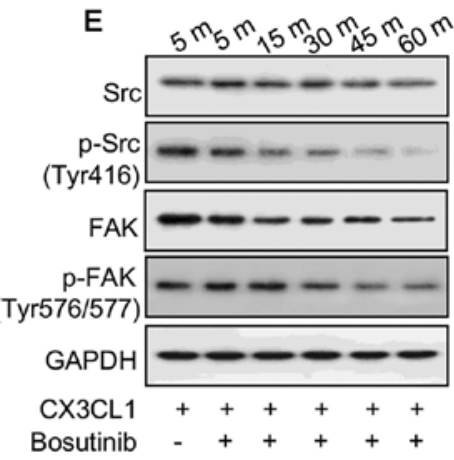

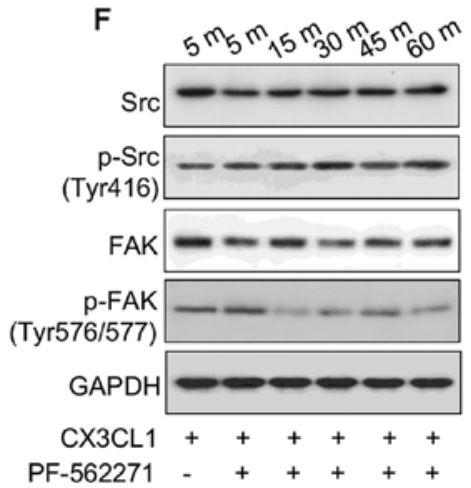

G

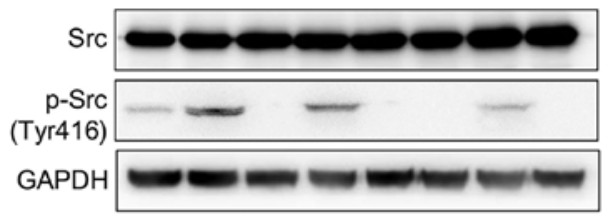

CX3CL1 (nM) $01000100100 \quad 0 \quad 100100$

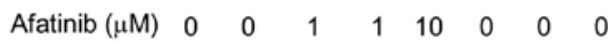

Bosutinib $(\mu \mathrm{M}) \quad 0 \quad 0 \quad 00000.250 .252$
$\mathrm{H}$

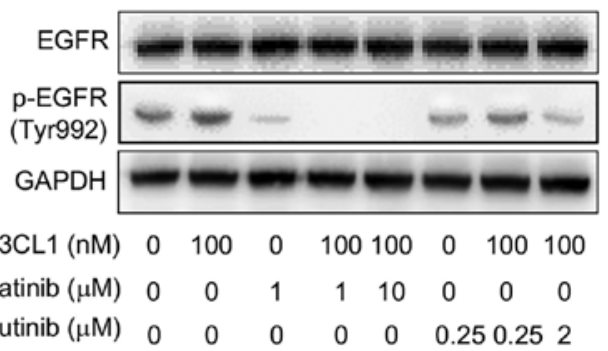

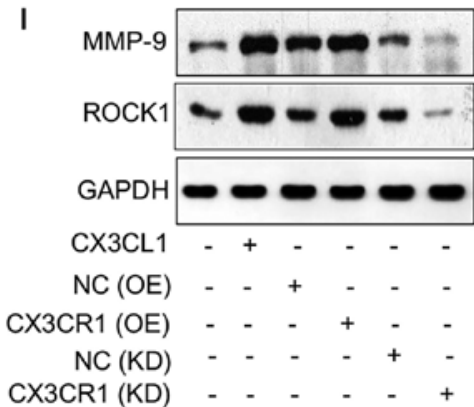

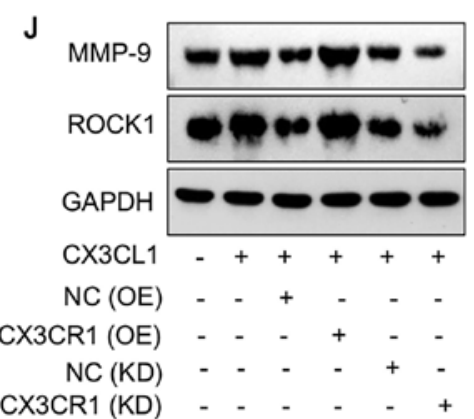

Figure 6. CX3CL1/CX3CR1 activates the Src/FAK pathway. (A) VCaP cells were treated either with or without CX3CL1 (100 nM) for 5, 15, 30, 45 or 60 min, and the expression of Src, p-Src (Tyr416), FAK and p-FAK (Tyr576/577) was determined by western blotting. (B) VCaP cells were treated either with or without CX3CL1 (100 nM) for 5, 15, 30, 45 or $60 \mathrm{~min}$, and the expression of EGFP, p-EGFR (Tyr992), PTK2B and p-PTK2B (Tyr402) was measured by western blotting. The expression of EGFR, p-EGFR (Tyr992), Src, p-Src (Tyr416), FAK and p-FAK (Tyr576/577) was measured by western blotting in CX3CL1-treated $(100 \mathrm{nM})$, stable CX3CR1-overexpressing or siRNA-induced CX3CR1-knockdown cells: (C) VCaP; (D) PC-3. PC-3 cells were pretreated with (E) bosutinib $(0.5 \mathrm{nM}$ for $3 \mathrm{~h}$ ) or with (F) PF-562271 ( $0.2 \mathrm{nM}$ for $0.5 \mathrm{~h})$, following which CX3CL1 was added to cells (100 nM for 5, 15, 30, 45 or $60 \mathrm{~min})$, and the expression of p-Src (Tyr416) and p-FAK (Tyr576/577) was examined. VCaP cells were pretreated with either afatinib (1 or $10 \mu \mathrm{M}$ for $4 \mathrm{~h})$ or bosutinib $(0.25$ or $2 \mu \mathrm{M}$ for $1 \mathrm{~h}$ ), following which CX3CL1 (100 nM) was added to cells (100 nM) for $30 \mathrm{~min}$, and the expression of (G) Src, p-Src (Tyr416), (H) EGFR and p-EGFR (Tyr992) were detected by western blotting. (I and J) The expression of MMP-9 and ROCK1 was measured by western blotting CX3CL1-treated (100 nM), stable CX3CR1-overexpressing or siRNA-induced CX3CR1-knockdown cells: (I) VCaP; (J) PC-3. Src, proto-oncogene tyrosine-protein kinase Src; FAK, focal adhesion kinase; EGFR, epidermal growth factor receptor; p, phosphorylated; OE, overexpression; NC, negative control; KD, knockdown; CON, control; CX3CL1, C-X3-C motif chemokine ligand 1; PTK2B, protein-tyrosine kinase 2- $\beta$; CX3CR1, C-X3-C motif chemokine receptor 1; MMP-9, matrix metalloproteinase-9; ROCK1, Rho-associated protein kinase 1. 

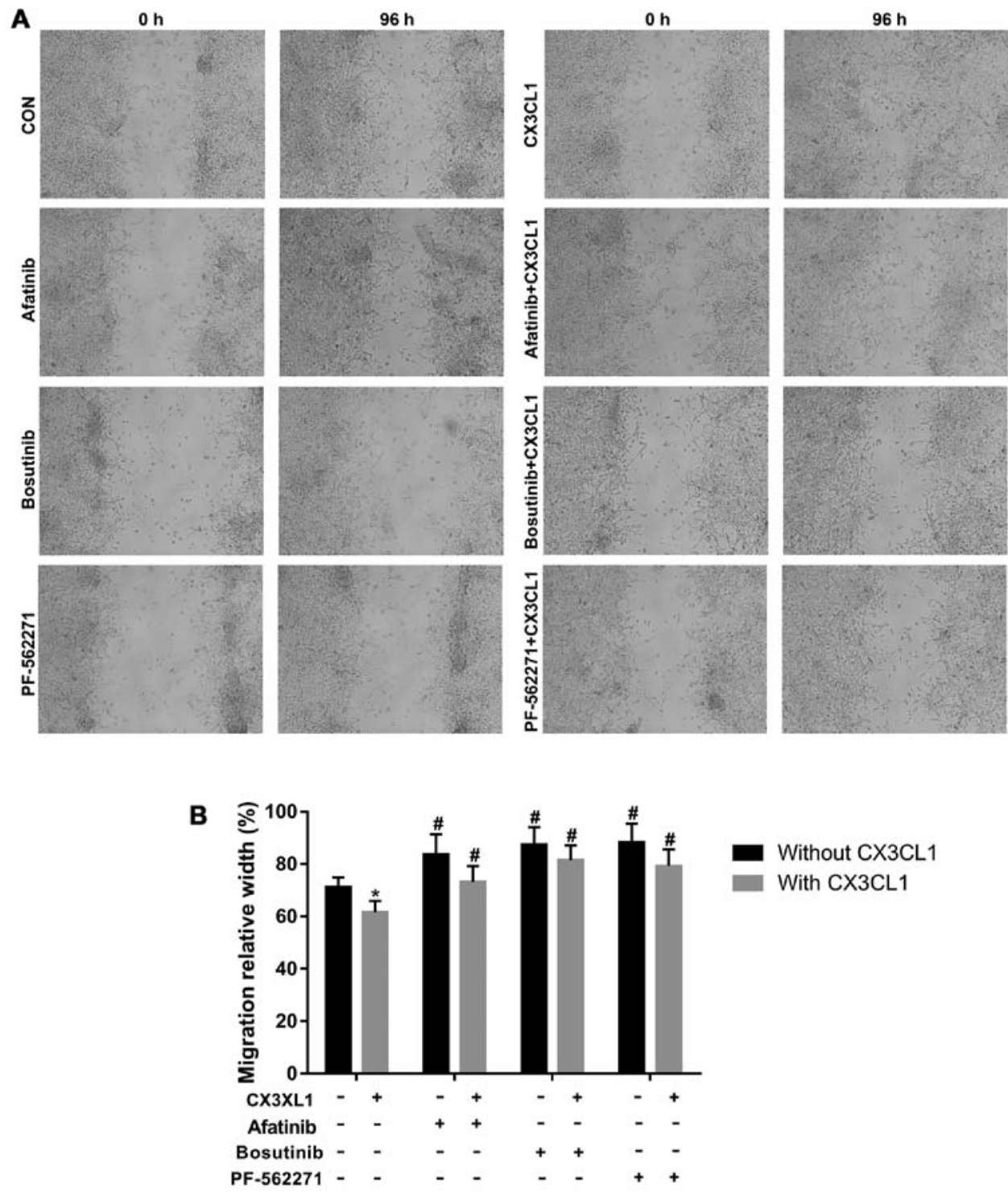

Figure 7. EGFR, Src and FAK inhibitors reverse cell migration induced by CX3CL1 in PC-3 cells. (A) Cell migration was measured by scratch wound assay. Representative images are presented (x200 magnification). (B) The results were summarized from three independent experiments. ${ }^{*} \mathrm{P}<0.05$ vs. without CX3CL1; ${ }^{\#}<<0.05$ vs. non-inhibitor-treated group. CX3CL1, C-X3-C motif chemokine ligand 1.

\section{A}

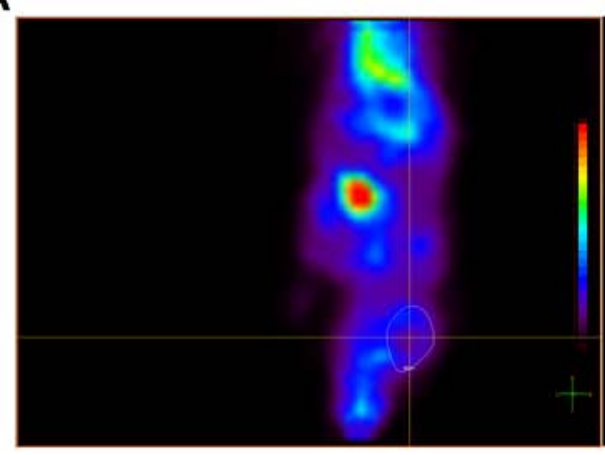

\section{B}

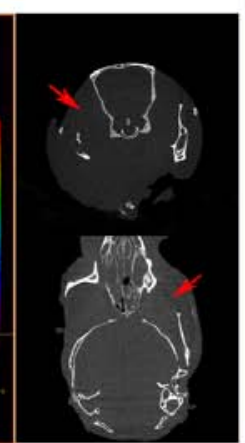

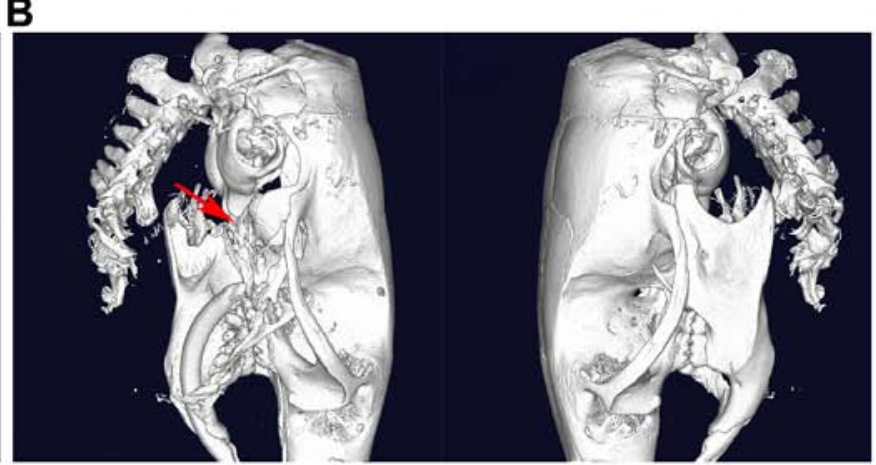

Figure 8. Prostate cancer metastasizes to maxillofacial bones. (A) There was a metastasis to the maxillofacial bones in one of the nine (11.11\%) mice in the control cell-injected group. Fluorodeoxyglucose was concentrated in the locality of the maxillofacial region. (B) A micro-computed tomography scan indicated local destruction (arrow) on the affected side of the maxillofacial bones, while on the opposite side the maxillofacial bones were intact.

to further verify the effects of the CX3CL/CX3CR1 axis on spine metastasis in other type of solid tumors. The present data suggest that CX3CL1/CX3CR1 may act as a potential target in metastatic prostate cancer therapy. 
A
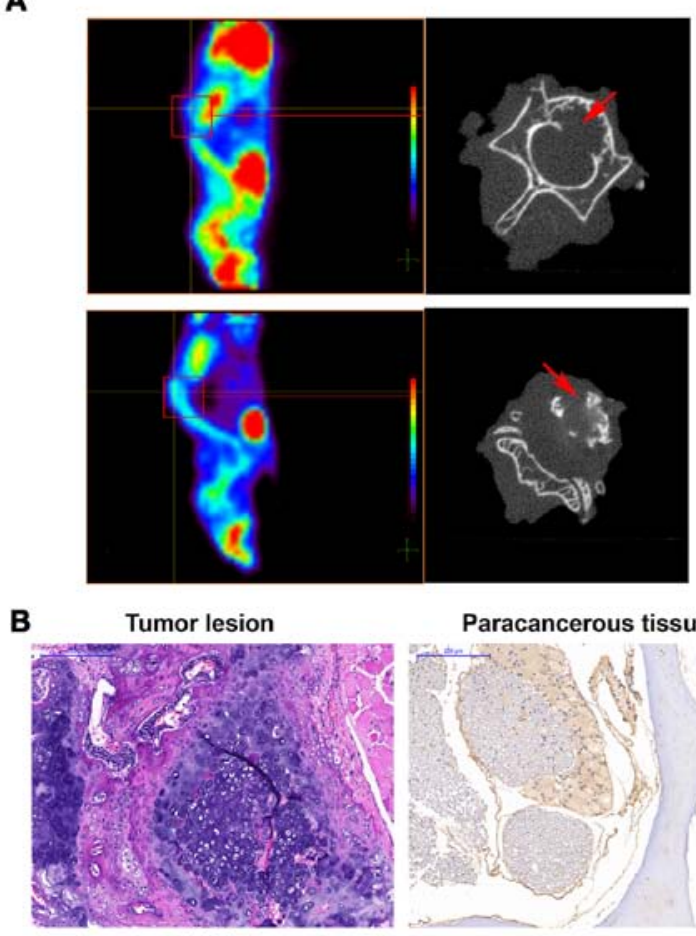

HE

Tumor lesion

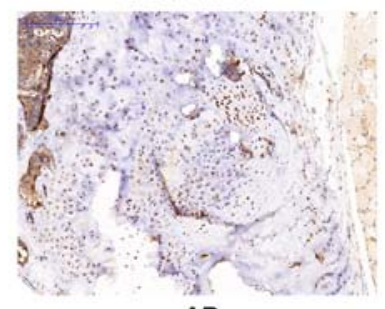

AR

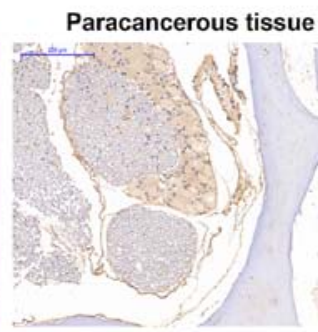

CX3CL1

Tumor lesion

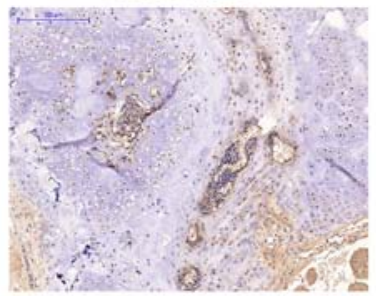

CX3CR1
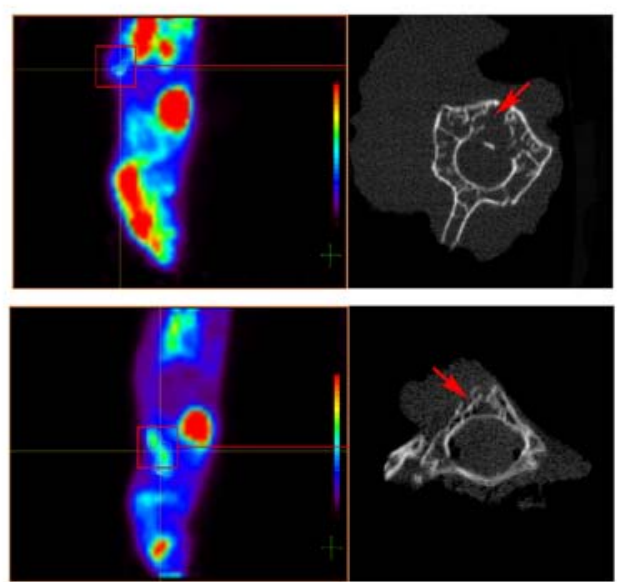

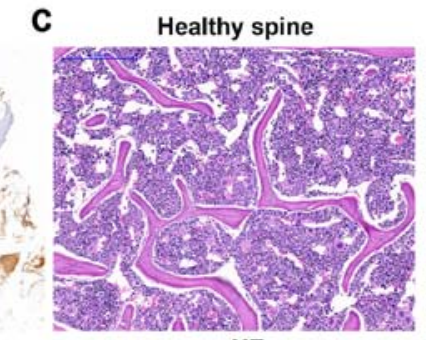

HE

Healthy spine

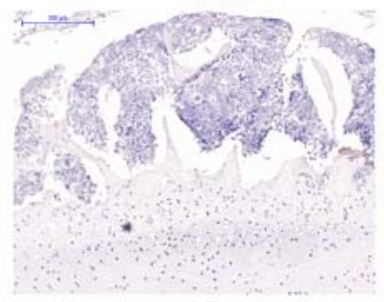

AR

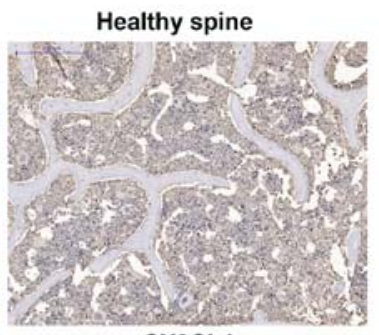

CX3CL1

Healthy spine

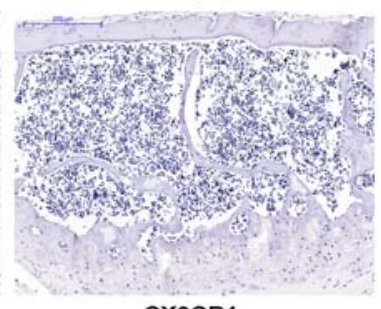

CX3CR1

Figure 9. CX3CL1/CX3CR1 facilitates the spinal metastasis of prostate cancer in vivo. (A) A total of 1x106 PC-3-control or PC3-CX3CR1-overexpressing cells were resuspended in $200 \mu 1$ serum-free medium and injected into the left ventricles of mice. After 6-8 weeks, the mice received a positron emission tomography scan. If a suspected spinal metastasis was found, the lesion underwent a further micro-CT scan. The micro-CT scans illustrated local destruction (arrow) in the affected vertebrae of the tumorigenic mice. H\&E staining and immunohistochemical staining of CX3CL1, CX3CR1 and AR is presented in (B) paracancerous tissues, tumor tissues and (C) healthy spine tissues (x100 magnification). CT, computed tomography; H\&E, hematoxylin and eosin; CX3CL1, C-X3-C motif chemokine ligand 1; CX3CR1, C-X3-C motif chemokine receptor 1.

\section{Acknowledgements}

Not applicable.

\section{Funding}

The present study was supported by the National Natural Science Foundation of China (grant nos. 81572629 and $81772855)$ and the China Postdoctoral Science Foundation (grant no. 2017M621362).

\section{Availability of data and materials}

All data generated or analyzed during this study are included in this published article.

\section{Authors' contributions}

PL and JD conceived and designed this study. PL, YL and HW conducted the experiments. PL and LJ analyzed and checked the data. SW collected and pretreated the clinical specimens. PL wrote the paper. JD supervised the whole experimental works and revised the manuscript. All authors have read and approved the final submitted manuscript.

\section{Ethics approval and consent to participate}

All the clinical samples were approved by the Ethics Committee of Zhongshan Hospital, Fudan University (Shanghai, China; no. Y2014-185). Informed consent was provided by the patients prior to surgery. The animal studies were approved by the Animal Ethics Committee of Zhongshan Hospital, Fudan University.

\section{Patient consent for publication}

Informed consent was provided by the patients prior to surgery.

\section{Competing interests}

The authors declare that they have no competing interests. 


\section{References}

1. Aaron AD: The management of cancer metastatic to bone JAMA 272: 1206-1209, 1994.

2. Dominguez DE, Lauper N, Velastegui A and Reynolds J: Surgical management of the spinal metastases. Rev Med Suisse 12 2168-2171, 2016 (In French)

3. Wu AS and Fourney DR: Evolution of treatment for metastatic spine disease. Neurosurg Clin N Am 15: 401-411, 2004.

4. Dushyanthen S, Cossigny DA and Quan GM: The osteoblastic and osteoclastic interactions in spinal metastases secondary to prostate cancer. Cancer Growth Metastasis 6: 61-80, 2013.

5. Choi D, Crockard A, Bunger C, Harms J, Kawahara N, Mazel C, Melcher R, Tomita K; Global Spine Tumor Study Group: Review of metastatic spine tumour classification and indications for surgery: the consensus statement of the Global Spine Tumour Study Group. Eur Spine J 19: 215-222, 2010.

6. Muralidharan A and Smith MT: Pathobiology and management of prostate cancer-induced bone pain: Recent insights and future treatments. Inflammopharmacology 21: 339-363, 2013.

7. Jemal A, Siegel R, Ward E, Murray T, Xu J and Thun MJ: Cancer statistics, 2007. CA Cancer J Clin 57: 43-66, 2007.

8. Przybyla BD, Shafirstein G, Vishal SJ, Dennis RA and Griffin RJ: Molecular changes in bone marrow, tumor and serum after conductive ablation of murine $4 \mathrm{~T} 1$ breast carcinoma. Int J Oncol 44: 600-608, 2014

9. Salazar N, Castellan M, Shirodkar SS and Lokeshwar BL: Chemokines and chemokine receptors as promoters of prostate cancer growth and progression. Crit Rev Eukaryot Gene Expr 23 77-91, 2013.

10. Liao YX, Zhou CH, Zeng H, Zuo DQ, Wang ZY, Yin F, Hua YQ and Cai ZD: The role of the CXCL12-CXCR4/CXCR7 axis in the progression and metastasis of bone sarcomas (Review). Int J Mol Med 32: 1239-1246, 2013.

11. Liu JF, Tsao YT and Hou CH: Fractalkine/CX3CL1 induced intercellular adhesion molecule-1-dependent tumor metastasis through the CX3CR1/PI3K/Akt/NF- $\kappa \mathrm{B}$ pathway in human osteosarcoma. Oncotarget 8: 54136-54148, 2016.

12. Tardáguila M, Mira E, García-Cabezas MA, Feijoo AM Quintela-Fandino M, Azcoitia I, Lira SA and Mañes S: CX3CL1 promotes breast cancer via transactivation of the EGF pathway. Cancer Res 73: 4461-4473, 2013.

13. Tang J, Xiao L, Cui R, Li D, Zheng X, Zhu L, Sun H, Pan Y, Du Y and Yu X: CX3CL1 increases invasiveness and metastasis by promoting epithelial-to-mesenchymal transition through the TACE/TGF- $\alpha$ /EGFR pathway in hypoxic androgen-independent prostate cancer cells. Oncol Rep 35: 1153-1162, 2016.

14. Ferretti E, Pistoia V and Corcione A: Role of fractalkine/ CX3CL1 and its receptor in the pathogenesis of inflammatory and malignant diseases with emphasis on B cell malignancies. Mediators Inflamm 2014: 480941, 2014

15. Shulby SA, Dolloff NG, Stearns ME, Meucci O and Fatatis A CX3CR1-fractalkine expression regulates cellular mechanisms involved in adhesion, migration, and survival of human prostate cancer cells. Cancer Res 64: 4693-4698, 2004.

16. Jamieson WL, Shimizu S, D'Ambrosio JA, Meucci O and Fatatis A: CX3CR1 is expressed by prostate epithelial cells and androgens regulate the levels of CX3CL1/fractalkine in the bone marrow: Potential role in prostate cancer bone tropism. Cancer Res 68: 1715-1722, 2008 .

17. Liu W, Bian C, Liang Y, Jiang L, Qian C and Dong J: CX3CL1: A potential chemokine widely involved in the process spinal metastases. Oncotarget 8: 15213-15219, 2017.

18. Wiemer AJ, Wernimont SA, Cung TD, Bennin DA, Beggs HE and Huttenlocher A: The focal adhesion kinase inhibitor PF-562,271 impairs primary $\mathrm{CD}^{+} \mathrm{T}$ cell activation. Biochem Pharmacol 86 : 770-781, 2013

19. Yu W, He X, Ni Y, Ngeow J and Eng C: Cowden syndrome-associated germline SDHD variants alter PTEN nuclear translocation through SRC-induced PTEN oxidation. Hum Mol Genet 24: $142-153,2015$.

20. Lee BY, Hochgräfe F, Lin HM, Castillo L, Wu J, Raftery MJ, Martin Shreeve S, Horvath LG and Daly RJ: Phosphoproteomic profiling identifies focal adhesion kinase as a mediator of docetaxel resistance in castrate-resistant prostate cancer. Mol Cancer Ther 13: 190-201, 2014.
21. Rabbani SA, Valentino ML, Arakelian A, Ali S and Boschelli F: SKI-606 (Bosutinib) blocks prostate cancer invasion, growth, and metastasis in vitro and in vivo through regulation of genes involved in cancer growth and skeletal metastasis. Mol Cancer Ther 9: 1147-1157, 2010.

22. Livak KJ and Schmittgen TD: Analysis of relative gene expression data using real-time quantitative PCR and the 2(-DeltaDeltaC(T)) method. Methods 25: 402-408, 2001.

23. Li HY, Cui XY, Wu W, Yu FY, Yao HR, Liu Q, Song EW and Chen JQ: Pyk2 and Src mediate signaling to CCL18-induced breast cancer metastasis. J Cell Biochem 115: 596-603, 2014.

24. Sarabia-Estrada R, Zadnik PL, Molina CA, Jimenez-Estrada I, Groves ML, Gokaslan ZL, Bydon A, Witham TF, Wolinsky JP and Sciubba DM: A rat model of metastatic spinal cord compression using human prostate adenocarcinoma: Histopathological and functional analysis. Spine J 13: 1597-1606, 2013.

25. Sun Y, Wang F, Sun X, Wang X, Zhang L and Li Y: CX3CR1 regulates osteoarthrosis chondrocyte proliferation and apoptosis via Wnt/beta-catenin signaling. Biomed Pharmacother 96: 1317-1323, 2017.

26. Wang H, Cai J, Du S, Guo Z, Xin B, Wang J, Wei W and Shen X Fractalkine/CX3CR1 induces apoptosis resistance and proliferation through the activation of the $\mathrm{AKT} / \mathrm{NF}-\kappa \mathrm{B}$ cascade in pancreatic cancer cells. Cell Biochem Funct 35: 315-326, 2017.

27. Luo W, Lin Y, Meng S, Guo Y, Zhang J and Zhang W: miRNA-296-3p modulates chemosensitivity of lung cancer cells by targeting CX3CR1. Am J Transl Res 8: 1848-1856, 2016.

28. Yao X, Qi L, Chen X, Du J, Zhang Z and Liu S: Expression of CX3CR1 associates with cellular migration, metastasis, and prognosis in human clear cell renal cell carcinoma. Urol Oncol 32: 162-170, 2014.

29. Xu Z, Zheng X, Yang L, Liu F, Zhang E, Duan W, Bai S, Safdar J, $\mathrm{Li} Z$ and Sun C: Chemokine receptor 7 promotes tumor migration and invasiveness via the RhoA/ROCK pathway in metastatic squamous cell carcinoma of the head and neck. Oncol Rep 33: 849-855, 2015

30. Lv Z, Hu M, Ren X, Fan M, Zhen J, Chen L, Lin J, Ding N, Wang $\mathrm{Q}$ and Wang R: Fyn mediates high glucose-induced actin cytoskeleton reorganization of podocytes via promoting ROCK activation in vitro. J Diabetes Res 2016: 5671803, 2016.

31. Hamaguchi $M$, Yamagata S, Thant AA, Xiao H, Iwata $H$, Mazaki $\mathrm{T}$ and Hanafusa $\mathrm{H}$ : Augmentation of metalloproteinase (gelatinase) activity secreted from Rous sarcoma virus-infected cells correlates with transforming activity of src. Oncogene 10: 1037-1043, 1995

32. Hunter T: A tail of two src's: Mutatis mutandis. Cell 49: 1-4, 1987.

33. Parsons JT, Martin KH, Slack JK, Taylor JM and Weed SA: Focal adhesion kinase: A regulator of focal adhesion dynamics and cell movement. Oncogene 19: 5606-5613, 2000.

34. Figel S and Gelman IH: Focal adhesion kinase controls prostate cancer progression via intrinsic kinase and scaffolding functions. Anticancer Agents Med Chem 11: 607-616, 2011.

35. Rentala S, Chintala R, Guda M, Chintala M, Komarraju AL and Mangamoori LN: Atorvastatin inhibited Rho-associated kinase 1 (ROCK1) and focal adhesion kinase (FAK) mediated adhesion and differentiation of $\mathrm{CD} 133{ }^{+} \mathrm{CD} 44^{+}$prostate cancer stem cells. Biochem Biophys Res Commun 441: 586-592, 2013.

36. Schlaepfer DD, Hanks SK, Hunter T and van der Geer P: Integrin-mediated signal transduction linked to Ras pathway by GRB2 binding to focal adhesion kinase. Nature 372: 786-791, 1994.

37. Jin JK, Dayyani F and Gallick GE: Steps in prostate cancer progression that lead to bone metastasis. Int J Cancer 128: 2545-2561, 2011

This work is licensed under a Creative Commons Attribution-NonCommercial-NoDerivatives 4.0 International (CC BY-NC-ND 4.0) License. 